\title{
Valores de la cultura organizacional y su relación con el engagement de los empleados: Estudio exploratorio en una organización de salud
}

\author{
Grueso-Hinestroza, M. ${ }^{1}$; González-Rodríguez, J. ${ }^{1}$ y Rey- \\ Sarmiento, C. ${ }^{2}$ \\ ${ }^{1}$ Universidad del Rosario, Escuela de Administración, Calle 12C \# 6-25, Bogotá, Colombia \\ ${ }^{2}$ Fundación Universitaria Sanitas, Facultad de Medicina, Carrera 7 No. 173 - 64, Bogotá, Colombia \\ * Autor para correspondencia. Email: merlin.grueso@urosario.edu.co
}

Recibido: 7 de febrero de 2014

Aceptado: 15 de abril de 2014

\begin{abstract}
In several models of healthy organizations has been postulated that organizational culture is a powerful predictor of employees wellbeing. From the above, this article seeks to demonstrate that relationship. To achieve this purpose, a questionnaire measuring five cultural values and Utrecht Work Engagement Scale _UWES - was administered. The sample of the study consisted of employees of a company that provides health services in Bogota (Colombia). The results show that the values of the organizational culture are predictors of employee engagement, although differently.
\end{abstract}

Keywords: Cultural values, Hofstede's model, Engagement, Healthy organizations, Well being.

\section{Resumen}

En diferentes modelos sobre organizaciones saludables se ha postulado que la cultura organizacional es un potente predictor de las condiciones de bienestar de los empleados. En función de lo anterior, el presente artículo busca demostrar tal relación. Para lograr dicho propósito, se administró un cuestionario que medía cinco valores culturales y la escala de engagement Utrech Work Engagement Scale -UWES- La muestra del estudio estuvo constituida por trabajadores de una empresa prestadora de servicios de salud de Bogotá 
Invest. pens. crit.

Vol. 2, No. 3, enero- abril 2014.

pp. $77-91$

(Colombia). Los resultados obtenidos señalan que los valores de la cultura organizacional son predictores del engagement de los empleados, aunque de manera diferenciada.

Palabras clave: valores culturales, modelo de Hofstede, engagement, organizaciones saludables, bienestar.

\section{Introducción}

Es claro que el bienestar de los trabajadores constituye un tema altamente relevante para las naciones, empresarios, académicos y sociedad en general, por tal razón, el presente artículo busca explicar la relación que existe entre los valores de la cultura organizacional y el bienestar de los trabajadores, utilizando como referente el modelo de organización saludable MIOS (Grueso y Rey, 2013). En función de lo anterior, el artículo inicia con un marco teórico, en el que se describen los conceptos que dan apoyo al diseño metodológico y al análisis de los resultados. La segunda parte compila aspectos relativos al método: tipo de estudio, participantes, instrumentos y procedimiento. En el tercer y cuarto apartado se describen los resultados de la investigación realizada y el análisis de los mismos. El artículo finaliza con unas conclusiones y limitaciones en torno al estudio.

\section{Organizaciones saludables}

El interés por la salud y el bienestar en el contexto de las organizaciones ha recibido especial atención en los últimos años. En diferentes ámbitos se ha venido discutiendo acerca de los mecanismos asociados con la promoción de la salud y el bienestar de los empleados tanto desde la perspectiva individual como organizacional, entre los que se encuentra la noción de organización saludable.

Las organizaciones saludables pueden se definidas como aquellas que gozan "de un estado de bienestar en función de sus factores relacionales y estructurales, tanto de carácter interno como externo" (Grueso y Rey, 2013, p. 634). En su propuesta Lowe (2004) destaca que las organizaciones saludables son aquellas donde "la cultura, el clima y las prácticas, crean un entorno que promueve la salud y seguridad del empleado así como la salud y seguridad organizacional" (p. 8). Acosta, Salanova y Llorens (2011) por su parte indican que las organizaciones saludables tienen tres componentes clave que interactúan entre sí: recursos y prácticas organizacionales saludables, empleados saludables y resultados organizacionales saludables.

Indistintamente de los fundamentos teóricos que subyacen a las anteriores aproximaciones sobre organizaciones saludables, estas tienen en común, que destacan la cultura organizacional como un factor determinante del bienestar de los grupos de interés, incluidos los empleados. Así por ejemplo Lowe (2004) defiende que la cultura organizacional se encuentra asociada con la

promoción de la salud y la seguridad tanto de los empleados como de la organización. Salanova 
Invest. pens. crit.

Vol. 2, No. 3, enero- abril 2014.

pp. $77-91$

(2009) a su vez señala que las organizaciones saludables cuentan con unos recursos estructurales tanto organizacionales como de tarea, que determinan la generación de resultados organizacionales saludables; en este contexto, la cultura organizacional constituye un recurso organizacional estructural que en conjunción con las prácticas directivas y de gestión de recursos humanos, determina el bienestar de los trabajadores, de los clientes y de la sociedad. El modelo MIOS (Grueso y Rey, 2013) también defiende que la cultura organizacional es un factor organizacional estructural que junto con los procesos y prácticas saludables, la estructura organizacional y la estrategia, predice el bienestar de los trabajadores y en general, el bienestar de los grupos de interés de las organizaciones.

\section{Cultura Organizacional}

Hofstede (1991) define la cultura organizacional como "la programación colectiva de la mente que distingue los miembros de una organización de otra" (p. 180) y añade además que se caracteriza por ser "holística, históricamente determinada, relacionada con el estudio de rituales y símbolos, socialmente construida, suave y difícil de cambiar" (p. 180). Se trata de un concepto que ha recibido atención durante las últimas décadas y con base en los desarrollos alcanzados, se ha logrado cierto grado de consenso sobre sus principales componentes: valores, ritos, mitos, leyendas (Deal y Kennedy, 1982; Pettigrew, 1979; Schein, 1992).

En torno de la cultura Hofstede (1979) propone un modelo de valores culturales, mediante el que recoge problemas comunes y los explica en diferentes contextos, incluido el organizacional: distancia al poder, masculinidad-feminidad, evitación de la incertidumbre y colectivismoindividualismo; la quinta dimensión incorporada posteriormente al modelo por Hofstede y Bond (1988), se denomina orientación de corto plazo-largo plazo. Para Hofstede (1991), las organizaciones con alta distancia al poder como valor organizacional, se decantan por la centralización de los procesos y la toma de decisiones, en su defecto, las organizaciones con baja distancia al poder se caracterizan por la descentralización de los procesos y la creación de un ambiente de participación y democracia.

En el modelo de valores culturales, Hofstede (1991) plantea que las organizaciones donde se experimentan valores orientados hacia la feminidad, priman acciones orientadas hacia la igualdad, la solidaridad y la calidad de vida; cuando los valores predominantes se encuentran

orientados hacia la masculinidad, las organizaciones suelen establecer formas de trato diferenciado entre el personal.

La evitación a la incertidumbre se constituye en otra dimensión del modelo de valores de la cultura desarrollado por Hofstede (1991), así, los contextos con baja evitación de la incertidumbre se caracterizan por aceptar y promover la diversidad organizacional, la integración de minorías y el respeto de los derechos de las personas; en contraste, los contextos en los que la evitación a la incertidumbre es alta, la diversidad y la integración de minorías no constituye en 
Invest. pens. crit.

Vol. 2, No. 3, enero- abril 2014.

pp. $77-91$

un tema de interés para la organización. Así mismo Hofstede (1991) señala que en los contextos donde priman valores colectivistas, prevalecen las ideologías sobre la igualdad y los intereses grupales en contraposición a los contextos con clara orientación hacia el individualismo, donde prevalecen los intereses individuales.

Finalmente y en función del modelo de valores culturales de Hofstede (1991), se plantea que las organizaciones donde predomina la orientación de largo plazo como valor, las tradiciones organizacionales se suelen adaptar al contexto en contraposición con culturas con orientación de corto plazo, caracterizadas por tener una concepción limitada de la inversión en la organización y donde se esperan resultados inmediatos.

Los planteamientos en torno al concepto de cultura organizacional desarrollados por Hofstede (1991) se circunscriben al enfoque socio-cultural (Lucas y García, 2002). Dicho enfoque señala que la cultura es el resultado de la asimilación de valores y patrones de comportamiento por parte de los miembros de una organización que tienen efecto en el contexto del trabajo (Hofstede, 1991). Otros autores complementan que la cultura organizacional determina aspectos procedimentales y operativos de las organizaciones (Kopelman, Brief y Guzzo, 1990; Rhody y Tang, 1995).

Y si bien el modelo de Hofstede (1979) ha recibido críticas (Klasing, 2013), se ha empleado en diferentes estudios, especialmente en el ámbito de los negocios internacionales (Leung, Bhagat, Buchan, Erez y Gibson, 2005). Este modelo también ha sido utilizado para analizar aspectos como la sensibilidad de las organizaciones en términos de equidad en el trato (Wheeler, 2002), el avance profesional de las mujeres en las organizaciones (Badjo y Dickson, 2001), en relación con la adopción de prácticas organizacionales saludables (Grueso, Rey y González, 2012), entre otros estudios.

El modelo de Hofstede (1979) también ha sido empleado para analizar su poder predictivo respecto del bienestar de los trabajadores. En este sentido, Genkova (2012) llevó a cabo un estudio en Bulgaria, Alemania, Francia y China, empleando como referente parte del modelo de Hofstede (1979): individualismo y colectivismo. Los resultados obtenidos en el estudio señalan que los patrones culturales predicen parcialmente el bienestar de los trabajadores a manera de satisfacción subjetiva.

\section{Engagement}

El engagement es definido como un "un estado mental positivo relacionado con el trabajo, caracterizado por vigor, dedicación y absorción” (Schaufeli, Salanova, González-Romá y Bakker, 2002, p. 74). En la literatura académica se ha defendido que el engagement constituye un constructo teóricamente opuesto al burnout (Salanova y Schaufeli, 2004), así, mientras el burnout implica altos niveles de cinismo, agotamiento, despersonalización e ineficacia laboral, el 
Invest. pens. crit.

Vol. 2, No. 3, enero- abril 2014.

pp. $77-91$

engagement implica elevados niveles de dedicación, vigor y absorción por parte del trabajador (Spontón y colaboradores, 2012).

En cuanto a las dimensiones que conforman el engagement, Schaufeli y colaboradores (2002) señalan que el vigor "se caracteriza por altos niveles de energía y resistencia mental mientras se trabaja, el deseo de invertir esfuerzos en el trabajo que se está realizando, incluso cuando aparecen dificultades en el camino" (p. 74). Así mismo, estos autores plantean que la dedicación "denota la alta implicación laboral, junto con la manifestación de un sentimiento de significación, entusiasmo, inspiración, orgullo y reto por el trabajo" (p. 74). Por último Schaufeli y colaboradores (2002) ponen de manifiesto que la absorción "ocurre cuando se está totalmente concentrado en el trabajo, mientras se experimenta que el tiempo 'pasa volando' y se tienen dificultades de desconectar de lo que se está haciendo debido a las fuertes dosis de disfrute y concentración experimentadas" (p. 74).

Existe evidencia empírica en torno de la relación que existe entre el engagement y factores organizacionales y personales, así por ejemplo Scanlan, Meredith y Poncen (2013) identificaron que las prácticas de balance trabajo/familia, la compensación en términos de reconocimiento y prestigio, de satisfacción personal y esfuerzo, tienen una relación positiva con el engagement de los trabajadores.

Las prácticas organizacionales saludables también han sido identificadas como potentes predictores del engagement de los empleados. En este sentido Acosta, Salanova y Llorens (2011) evidenciaron que el engagement en equipos de trabajo es producto de la adopción de prácticas organizacionales saludables, actuando como variable mediadora la confianza en la organización. De igual forma, Alfes, Shantz y Truss (2012) llevaron a cabo un estudio en el que analizaban la relación entre bienestar y prácticas de recursos humanos. Los resultados obtenidos señalan que los empleados que perciben positivamente las prácticas de recursos humanos, experimentan mayor bienestar, siempre que exista confianza hacia la organización. Finalmente, existe evidencia que el entorno físico, cultural y sistémico de trabajo, aspectos específicos del trabajo al igual que las interacciones con colegas, clientes y supervisores, son potentes predictores del bienestar subjetivo en los trabajadores (Shier y Graham, 2011).

No obstante se ha demostrado que las condiciones organizacionales tienen efecto en el bienestar de los empleados (Acosta, Salanova y Llorens, 2011; Alfes, Shantz y Truss , 2012; Scanlan, Meredith y Poncen, 2013) y se ha puesto de manifiesto la existencia de relaciones estadísticamente significativas entre la cultura organizacional y el bienestar y/o el engagement de los trabajadores (Calderón, Murillo y Torres, 2003; Chang y Lu, 2007; Moreno y Báez, 2010; Roozeboom, Houtman y Van den Bossche, 2008; Sarangi y Srivastava, 2012), no existe un cuerpo de investigación que explique el engagement de los empleados usando como referencia el modelo de valores culturales de Hofstede (1979), razón por la cual se plantea el presente artículo de investigación. 
Invest. pens. crit.

Vol. 2, No. 3, enero- abril 2014.

pp. $77-91$

\section{Materiales y método}

Tipo de estudio

La investigación realizada corresponde a un estudio de carácter descriptivo-correlacional.

\section{$\underline{\text { Participantes }}$}

La población para el estudio estuvo constituida por los empleados de una empresa de salud de carácter privado que ofrece sus servicios en la ciudad de Bogotá. La muestra para el estudio, estuvo constituida por 62 trabajadores de la empresa objeto de análisis. Con el fin de garantizar la representatividad en la participación, se realizó un muestreo estratificado.

\section{$\underline{\text { Instrumentos }}$}

Se administró a los empleados un cuestionario que contenía dos escalas de medida y un conjunto de variables sociodemográficas así:

\section{a. $\quad$ alores culturales}

Para evaluar los valores organizacionales se empleó una escala (Grueso, 2010; Grueso, Rey y González, 2012) inspirada en el Modelo de Hofstede (1979). La escala contiene 35 reactivos y 5 dimensiones denominadas así: distancia al poder, masculinidad-feminidad, colectivismoindividualismo, tolerancia a la incertidumbre y orientación corto plazo-largo plazo.

Distancia al poder: Un alto índice de distancia al poder se encuentra aparejado con la inequidad, centralización, autoritarismo y división de clases; un bajo índice de distancia al poder por el contrario, implica la búsqueda de la equidad, la descentralización y la participación de las personas (1: alto - 5: bajo).

Masculinidad - Feminidad: Un alto índice de masculinidad se encuentra asociado con segregación ocupacional, presencia de estereotipos de género y diferencias en el estatus entre el hombre y la mujer; en contraposición, un alto índice de feminidad se asocia con la integración profesional entre los sexos, ausencia de estereotipos de género, trato equitativo hacia hombres y mujeres (1: masculinidad - 5: feminidad).

Tolerancia a la incertidumbre: ${ }^{1}$ Un bajo índice de tolerancia a la incertidumbre es expresado mediante actitudes negativas hacia la diversidad y falta de respeto por los derechos humanos. Por el contrario, un alto índice de tolerancia a la incertidumbre se expresa mediante actitudes positivas hacia la diversidad, la integración de minorías y el respeto por las personas (1: baja tolerancia 5: alta tolerancia).

\footnotetext{
${ }^{1}$ En el modelo de Hofstede (1979) esta dimensión se denomina Evitación de la incertidumbre
} 
Invest. pens. crit.

Vol. 2, No. 3, enero- abril 2014.

pp. $77-91$

Colectivismo - Individualismo: Los altos índices de colectivismo se encuentran asociados a la cohesión social, la valoración de las relaciones interpersonales, el establecimiento de relaciones morales entre los miembros de un colectivo y alta sensibilidad hacia la equidad. Por el contrario, un alto índice de individualismo se caracteriza por la valoración de la independencia, el desempeño, los logros individuales y por la ausencia de sensibilidad hacia la equidad (1: individualismo - 5: colectivismo).

Orientación corto plazo - largo plazo: La orientación hacia el corto plazo se encuentra aparejada con el énfasis en el estatus, la espera de resultados inmediatos y un respeto por la tradición; por el contrario, la orientación de largo plazo se encuentra asociada con la perseverancia, la espera de resultados a medio y largo plazo y la adaptabilidad a las condiciones del entorno (1: orientación al corto plazo - 5: orientación al largo plazo).

En las investigaciones donde se ha empleado esta escala (Grueso, 2010; Grueso, Rey y González, 2012), se han obtenido propiedades psicométricas satisfactorias y los coeficientes de fiabilidad reportados han sido superiores a 0.80 , lo cual, de acuerdo con Nunnally (1978) es apropiado.

\section{b. $\quad$ Engagement versión corta}

La versión corta de la escala de Engagement (Salanova y Schaufeli, 2004) cuenta con nueve reactivos y tres dimensiones. Cuenta con 6 anclajes de respuesta que van de nunca (1) a siempre (6). Esta escala ha sido empleada estudios previos y las propiedades psicométricas reportadas han sido satisfactorias (Schaufeli, Bakker y Salanova, 2006)

\section{Procedimiento}

Para dar inicio al trabajo de campo, se solicitó la autorización a la gerencia de la empresa, quienes a su vez tramitaron la solicitud ante el Comité de Ética. Los instrumentos a emplear y el alcance de la investigación, fue evaluado y en función de ello se autorizó el desarrollo del estudio. El siguiente paso fue la determinación de la muestra para el estudio y la invitación a los participantes. Quienes aceptaron voluntariamente a participar firmaron un consentimiento informado. Posterior a la firma del consentimiento informado, se administraron los cuestionarios garantizando la absoluta confidencialidad de la información. En cuanto a los datos, estos fueron procesados con el paquete estadístico SPSS versión 20, para realizar análisis factoriales, de fiabilidad, así como también para calcular coeficientes de correlación y realizar una regresión lineal.

\section{Resultados}

\section{Variables sociodemográficas}

Sexo: El 27.4\% de los participantes eran hombres. El 72.6\% de los participantes eran mujeres. En cuanto a la edad, y de acuerdo con los resultados del estudio, el 6\% de los participantes tenían 
Invest. pens. crit.

Vol. 2, No. 3, enero- abril 2014.

pp. $77-91$

menos de 25 años, el 31\% tenían edades que oscilaban entre 25 y 35 años. Un 35\% de los participantes contaban con edades entre 36 y 45 años. El 24\% de la muestra del estudio tenían una edad entre 46 y 55 años y solo el 3\% de la muestra contaba con más de 55 años. El 58\% de los participantes en el estudio tenían menos de 5 años de antigüedad en el cargo. El 6\% tenían entre 5.1 y 10 años de antigüedad en el cargo, el 3\% tenían entre 10.1 y 15 años de antigüedad en el cargo. Complementariamente, el 9\% de los participantes tenían entre 15.1 y 20 años de antigüedad en el cargo, el 6\% entre 20.1 y 25 años de antigüedad, el 3\% entre 25.1 y 30 años y el $2 \%$ de los participantes contaban con más de 30 años de antigüedad en el cargo. Finalmente y en relación con el nivel del cargo, se identifica que el 11\% de los participantes desempeñan cargos de nivel operativo, el 31\% desempeñan cargos de nivel auxiliar, el 11\% se encuentran en cargos de nivel asistente, 5\% desempeñan cargos de nivel técnico, el 13\% desempeñan cargos de nivel profesional, el 11\% se encuentran en cargos de nivel profesional especializado. De igual forma, un $6 \%$ de los participantes de la muestra desempeñan cargos de coordinación, 2\% de nivel directivo y un $10 \%$ de los participantes no reportan el nivel de cargo.

\section{Propiedades psicométricas de las escalas}

Para probar la hipótesis del estudio, se administraron dos escalas de medida y se analizaron sus propiedades psicométricas, en términos de validez y fiabilidad. Para analizar la validez de las escalas se realizó un análisis factorial exploratorio; los ítems que saturaron a cada factor con valores superiores a .40 fueron considerados como parte del mismo. La escala de valores culturales conservó la estructura factorial esperada en 5 dimensiones. De otra parte, el análisis factorial realizado con la escala de Engagement de Schaufeli, Bakker y Salanova (2006) llevó a concluir que la estructura factorial del constructo correspondía, en este estudio, a un único factor.

Una vez identificados los factores para cada escala, se procedió a calcular el coeficiente de fiabilidad para cada uno de ellos. La Tabla 1 describe las escalas, el número de factores, el valor medio obtenido y los coeficientes de fiabilidad. Tal como se observa, todos los factores que componen las escalas reflejaron coeficientes de fiabilidad entre .80 y .90 . De acuerdo con Nunnally (1978) coeficientes de fiabilidad superiores a .70 son considerados como adecuados. 
Invest. pens. crit.

Vol. 2, No. 3, enero- abril 2014.

pp. $77-91$

Tabla 1. Coeficientes de fiabilidad de las escalas

\begin{tabular}{lccc}
\hline \multicolumn{1}{c}{ Escala } & \# Items & Media & $\begin{array}{c}\text { Alpha de } \\
\text { Cronbach }\end{array}$ \\
\hline Valores Culturales & & & \\
Distancia al poder & 7 & 3.4 & .86 \\
Masculinidad-Feminidad & 7 & 3.7 & .83 \\
Colectivismo-Individualismo & 8 & 3.7 & .89 \\
Tolerancia a la incertidumbre & 8 & 3.6 & .86 \\
Orientación corto plazo-largo plazo & 5 & 3.8 & .80 \\
Engagement & & & .88 \\
Engagement & 9 & 5.0 & \\
\hline
\end{tabular}

Fuente: Autores

En cuanto al valor promedio de las escalas estudiadas, se observa que los valores culturales oscilan entre 3.8 y 3.4, siendo el valor más bajo el correspondiente a la variable distancia al poder. El valor promedio de la escala de engagement fue de 5.0 sobre 6.0.

\section{$\underline{\text { Correlaciones }}$}

Todos los valores de la cultura organizacional analizados se encuentran asociados significativamente con el engagement de los trabajadores (Tabla 2).

Tabla 2. Correlaciones (r de Pearson)

\begin{tabular}{lllllll}
\hline \multicolumn{1}{c}{ Variables } & 1 & 2 & 3 & 4 & 5 & 6 \\
\hline 1. Distancia al poder & - & & & & \\
2. Masculinidad-Feminidad & & & & & \\
& $.853^{* *}$ & - & & & \\
3. Colectivismo-Individualismo & $(\mathrm{p}=.000)$ & & & & \\
& $.923^{* *}$ & $.876^{* *}$ & - & & \\
4. Tolerancia a la incertidumbre & $(\mathrm{p}=.000)$ & $(\mathrm{p}=.000)$ & & & \\
& $.879^{* *}$ & $.866^{* *}$ & $.895^{* *}$ & - & \\
5. Orientación corto plazo-largo plazo & $(\mathrm{p}=.000)$ & $(\mathrm{p}=.000)$ & $(\mathrm{p}=.000)$ & & \\
& $.802^{* *}$ & $.771^{* *}$ & $.851^{* *}$ & $.824^{* *}$ & - & - \\
& $(\mathrm{p}=.000)$ & $(\mathrm{p}=.000)$ & $(\mathrm{p}=.000)$ & $(\mathrm{p}=.000)$ & & \\
6. Engagement & $.373^{* *}$ & $.440^{* *}$ & $.439^{* *}$ & $.449^{* *}$ & $.352^{* *}$ & - \\
& $(\mathrm{p}=.003)$ & $(\mathrm{p}=.000)$ & $(\mathrm{p}=.000)$ & $(\mathrm{p}=.000)$ & $(\mathrm{p}=.005)$ & \\
\hline
\end{tabular}

**. La correlación es significativa al nivel 0.01 (bilateral).

Fuente: Autores

El coeficiente de correlación entre el valor distancia al poder y engagement fue de .37 con una significación de $p=.01$. Significativa es la relación que existe entre el valor masculinidadfeminidad y el engagement de los trabajadores $(r=.44, p=.01)$. Similar coeficiente se obtuvo 
Invest. pens. crit.

Vol. 2, No. 3, enero- abril 2014.

pp. $77-91$

al analizar la relación entre el valor tolerancia a la incertidumbre y el engagement $(r=.44, p=$ .01) y un valor muy cercano se evidenció al analizar la relación entre el valor colectivismoindividualismo y el engagement $(r=.43, p=.01)$. El valor orientación corto plazo-largo plazo en función del engagement obtuvo un coeficiente de correlación de .35 con una significación de $p=.01$.

Para validar el poder predictivo de los valores de la cultura en función del engagement se realizó una regresión, empleando el método de pasos sucesivos (Tabla 3). De manera particular, los resultados indican que la tolerancia a la incertidumbre es el único valor que predice el engagement de los trabajadores. En contraste, los valores distancia al poder, masculinidad-feminidad, colectivismo-individualismo y orientación de corto plazo-largo plazo, no predicen el desarrollo del engagement de los trabajadores.

Tabla 3. Regresión

\begin{tabular}{cllll}
\hline Modelo & \multicolumn{2}{c}{ Variables introducidas } & Variables eliminadas & \multicolumn{1}{c}{ Método } \\
\hline \multirow{2}{*}{1} & Tolerancia & a & la & Por pasos (criterio: Prob. de F \\
& incertidumbre & & &
\end{tabular}

a. Variable dependiente: Engagement

Fuente: Autores

De acuerdo con el modelo de regresión (Tabla 4) el R calculado para la relación entre el valor tolerancia a la incertidumbre y engagement fue .449 y el $\mathrm{R}^{2}=.202$.

Tabla 4. Resumen del modelo

\begin{tabular}{|c|c|c|c|c|c|c|c|c|}
\hline \multirow[b]{2}{*}{ Modelo } & \multirow[b]{2}{*}{$\mathrm{R}$} & \multirow[b]{2}{*}{$\mathrm{R}^{2}$} & \multirow[b]{2}{*}{$\begin{array}{l}\text { Error típ. de } \\
\qquad \text { la } \\
\text { estimación }\end{array}$} & \multicolumn{5}{|c|}{ Estadísticos de cambio } \\
\hline & & & & Cambio en $\mathrm{R}^{2}$ & Cambio en F & gl1 & gl2 & $\begin{array}{c}\text { Sig. } \\
\text { Cambio } \\
\text { en F }\end{array}$ \\
\hline 1 & $.449^{a}$ & .202 & .77721 & .202 & 15.153 & 1 & 60 & .000 \\
\hline
\end{tabular}

a. Variables predictoras: (Constante), Tolerancia a la incertidumbre

Fuente: Autores

Finalmente el modelo de regresión que incorpora el valor Tolerancia a la incertidumbre y el engagement evidencia que la relación entre estas variables es estadísticamente significativa $(\mathrm{p}=.00)$, por tanto, es posible señalar que el valor tolerancia a la incertidumbre predice el engagement de los trabajadores, tal como se ilustra en la Tabla 5. 
Invest. pens. crit.

Vol. 2, No. 3, enero- abril 2014.

pp. $77-91$

Tabla 5. Análisis de Varianza Anova del Modelo

\begin{tabular}{|c|c|c|c|c|c|c|}
\hline & Modelo & $\begin{array}{l}\text { Suma de } \\
\text { cuadrados }\end{array}$ & $\mathrm{gl}$ & Media cuadrática & $\mathrm{F}$ & Sig. \\
\hline \multirow{3}{*}{1} & Regresión & 9.153 & 1 & 9.153 & 15.153 & $.000^{\mathrm{b}}$ \\
\hline & Residual & 36.243 & 60 & .604 & & \\
\hline & Total & 45.396 & 61 & & & \\
\hline
\end{tabular}

a. Variable dependiente: ENGAGEMENT

b. Variables predictoras: (Constante), TOLERANCIA A LA INCERTIDUMBRE

Fuente: Autores

\section{Discusión}

Los resultados obtenidos en la presente investigación, si bien no son concluyentes, permiten realizar una serie de consideraciones respecto de la relación que existe entre los valores de la cultura organizacional y el engagement de los empleados. Diferentes modelos de organizaciones saludables (Grueso y Rey, 2013; Lowe, 2004; Salanova, 2008) plantean que factores organizacionales como la cultura organizacional, son predictores del bienestar del trabajador. En estudios previos (Calderón, Murillo y Torres, 2003; Chang y Lu, 2007; Moreno y Báez, 2010; Roozeboom, Houtman y Van den Bossche, 2008; Sarangi y Srivastava, 2012) ya se había evidenciado tal relación, aunque empleando aproximaciones a la cultura organizacional distintas al modelo de Hofstede (1979). Los resultados del presente estudio permiten demostrar las relaciones previstas, aunque de manera parcial.

De acuerdo con los resultados obtenidos el valor organizacional tolerancia a la incertidumbre parece ser un buen predictor del engagement de los trabajadores, así, a mayor tolerancia a la incertidumbre, mayor engagement de los trabajadores. Esta relación tendría alguna explicación, en la medida en que la tolerancia a la incertidumbre implica que las organizaciones expresan actitudes positivas hacia la diversidad, la integración de minorías y el respeto por las personas. Un contexto laboral con tales características, debe tener consecuencias en términos de alta implicación laboral y deseos de invertir esfuerzos en el trabajo, en definitiva mayor engagement por parte de los trabajadores.

Los resultados del estudio llevan a concluir que otros los valores de la cultura organizacional (distancia al poder, masculinidad-feminidad, colectivismo-individualismo y orientación corto plazo-largo plazo), no son predictores del engagement de los trabajadores, aunque existían evidencias previas que sugerían la posibilidad de tal relación. Por ejemplo Sarangi y Srivastava (2012) en su estudio demostraron que el valor colectivismo- individualismo era un predictor del bienestar de los trabajadores. De igual forma Scanlan, Meredith y Poncen (2013) habían demostrado que existe una relación entre ciertos factores organizacionales y el engagement de los trabajadores. Así mismo, existían evidencias previas en las que se demostraba la existencia de 
Invest. pens. crit.

Vol. 2, No. 3, enero- abril 2014.

pp. $77-91$

una relación estadísticamente significativa entre la cultura organizacional y el engagement o el bienestar de los trabajadores (Calderón, Murillo y Torres, 2003; Chang y Lu, 2007; Moreno y Báez, 2010; Roozeboom, Houtman y Van den Bossche, 2008; Sarangi y Srivastava, 2012).

Estos resultados pueden ser explicados en alguna medida. Al parecer, no es cualquier tipo de valor cultural el que estaría asociado con el engagement de trabajadores. Es posible que sean valores que representen la existencia de una cultura de apoyo la que estaría generando el engagement en los trabajadores, tal como lo expresa Lowe (4004).

\section{Conclusiones}

El bienestar de los trabajadores y de otros grupos de interés es un tema de alta importancia tanto a nivel organizacional como social, pues más allá de responder a temas humanitarios y de sostenibilidad, las organizaciones saludables aseguran su permanencia al tener mejor un desempeño (Keller y Price, 2011).

En el presente estudio, se analizó la relación hipotetizada entre los valores de la cultura organizacional y el engagement de los trabajadores, con base en supuestos del modelo de organizaciones saludables de Grueso y Rey (2013). Los resultados entregan evidencia parcial de las relaciones entre las variables estudiadas y abre nuevos caminos para continuar investigando sobre el tema. Y si bien Hofstede (1991) planteaba que los valores culturales tienen efecto en el contexto del trabajo, valdría la pena revisar al emplear una escala que identifique valores organizacionales orientados al apoyo y el cuidado del otro, los resultados que se obtienen son diferentes. También sería pertinente incorporar en nuevos estudios una variable mediadora o moderadora de la relación entre la cultura organizacional y el engagement de los trabajadores, como pueden ser las prácticas organizacionales saludables, entre las que se pueden encontrar un conjunto de prácticas de recursos humanos, entre otras.

Finalmente, es claro que si una organización es saludable obtiene mejores resultados (Keller y Price, 2011). Pese a lo anterior, aún los gobiernos y las empresas no han comprendido las ventajas de crear entornos laborales saludables o no cuentan con las habilidades, herramientas, habilidades o conocimientos para crearlos (OMS, 2010). En este sentido, se hace necesario seguir investigando en torno a este tema.

Como es razonable, la investigación presenta algunas limitaciones. Por ejemplo, los participantes procedían de una única empresa y la muestra empleada en el estudio resulta ser limitada, a pesar que el muestreo fue de tipo estratificado para asegurar la representatividad de los participantes en la empresa. En consecuencia, futuras investigaciones deberán considerar muestras poblacionales más amplias.

En relación con las escalas de medida, es necesario realizar estudios con muestras más amplias en Colombia. Llama la atención la escala de engagement versión corta de Schaufeli, Bakker y 
Invest. pens. crit.

Vol. 2, No. 3, enero- abril 2014.

pp. $77-91$

Salanova (2006), pues si bien sus autores identifican la existencia de tres factores que le subyacen, en la presente investigación el análisis factorial muestra la existencia de uno.

\section{Agradecimientos}

A las Magister Adriana Ardila y Carolina Pineda, quienes contribuyeron a la recolección de los datos durante el trabajo de campo.

\section{Referencias}

Acosta, H., Salanova, M. y Llorens, S. (2011). ¿Cómo predicen las prácticas organizacionales el engagement en el trabajo en equipo?: El rol de la confianza organizacional. Ciencia \& Trabajo, 41, 125-134.

Alfes, K., Shantz, A. y Truss, C. (2012). The link between perceived HRM practices, performance and well-being: the moderating effect of trust in the employer. Human Resource Management Journal, 22, 409-427.

Badjo, L. y Dickson, M. (2002). Perceptions of organizational culture and women's advancement in organizations: A cross-cultural examination. Sex Roles, 45 (5/6), 399-414.

Calderón, G., Murillo, S. M. y Torres, K. Y. (2003). Cultura organizacional y bienestar laboral. Cuadernos de Administración, 16 (25), 109-137.

Chang, K. y Lu, L. (2007). Characteristics of organizational culture, stressors and wellbeing: The case of Taiwanese organizations. Journal of Managerial Psychology, 22 (6), 549-568.

Deal, T. E. y Kennedy, A. A. (1982). Corporate cultures: The rites and rituals of corporate life. Reading, M.A.: Addison Wesley.

Genkova, P. (2012). Cultural patterns and subjective culture as predictors of well-being: a crosscultural study. Psychology Research, 2 (3), 177-184.

Grueso, M. P. y Rey, C. F. (2013). Hacia la construcción de un modelo explicativo de organizaciones saludables. Revista de Ciencias Sociales, XIX (4), 625 - 638.

Grueso, M. P. (2010). Implementación de buenas prácticas de promoción de personal y su relación con valores de la cultura y el compromiso con la organización. Revista Innovar, 20 (36), 79-90.

Hofstede, G. (1991). Cultures and organizations: Software of the mind. Inglaterra: Mc-Graw Hill Book Company Europe

Hofstede, G. y Bond, M. (1988). The Confucius connection: from cultural roots to economic growth. Organizational Dynamics, 16 (4), 4-21. 
Invest. pens. crit.

Vol. 2, No. 3, enero- abril 2014.

pp. $77-91$

Hofstede, G. (1979). Value systems in forty countries: Interpretation, validation and consequences for theory. En L.H. Eckensburger (Ed), Cross-cultural contribution to psychology. Alemania: Swets and Zeitlinger B.V.

Keller, S. y Price, C. (2011). Organizational health: The ultimate competitive advantage. McKinsey Quarterly, [en línea] consultado el 10 de enero de 2014, http://www.mckinsey.com/insights/organization/organizational_health_the_ultimate _competitive_advantage.

Kopelman, R., Brief, A. y Guzzo, R. (1990). The role of climate and culture in productivity. En B. Schneider (Ed.) Organizational climate and culture. San Francisco: Jossey Bass Publishers.

Leung, K., Bhagat, R., Buchan, N., Erez, M. y Gibson, C. (2005). Culture and international business: recent advances and their implications for future research. Journal of International Business Studies, 36, 357-378.

Lowe, G. (2004). Healtby workplace strategies: creating change and achieving results. [en línea] consultado el 15 de enero de 2014, http://www.mentalhealthpromotion.net/resources/healthyworkplace-strategies.pdf

Lucas, M. A. y García, R. P. (2002). Sociología de las organizaciones. McGraw Hill, Madrid.

Nunnally, J. (1978). Psychometric theory (2a ed.). New York: McGraw Hill.

Organización Mundial de la Salud OMS (2010). Entornos laborales saludables: Fundamentos y modelo de la OMS. Contextualización, prácticas y literatura de apoyo. [en línea], consultado el 18 de enero de 2014, http://apps.who.int/iris/handle/10665/44466.

Pettigrew, A. (1979). On studying organizational cultures. Administrative Science Quarterly, 24 (4), $570-581$.

Rhody, J. D. y Tang, T. (1995). Learning from japanese transplants and american corporations. Public Personnel Management, 24, 19-32.

Salanova, M. (2008). Organizaciones saludables: Una aproximación desde la psicología positiva. En C. Vázquez y G. Hervás (Eds.), Psicología Positiva: Bases científicas del bienestar y la resiliencia, Alianza Editorial, Madrid.

Salanova, M. y Schaufeli, W. (2004). El Engagement de los empleados: Un reto emergente para la dirección de recursos humanos. Estudios financieros. Revista de trabajo y seguridad social: Comentarios, casos prácticos: recursos humanos, (261), 109-138.

Sarangi, S. y Srivastava, R. K. (2012). Impact of organizational culture and communication on employee engagement: An investigation of Indian private banks. South Asian Journal of Management, 19 (3), 18-33.

Scanlan, J. N., Meredith, P. \& Poulsen, A. A. (2013). Enhancing retention of occupational therapists working in mental health: Relationships between wellbeing at work and 
Invest. pens. crit.

Vol. 2, No. 3, enero- abril 2014.

pp. $77-91$

turnover intention. Australian Occupational Therapy Journal, 60 (6), 395-403, DOI: 10.1111/1440-1630.12074.

Schaufeli, W. B., Bakker, A. B. y Salanova, M. (2006). The measurement of work engagement with a short questionnaire. A cross-national study. Educational and Psychological Measurement, 66 (4), 701-716.

Schein, E. (1992). Organizational culture and leadership (2a ed). San Francisco: Jossey-Bass.

Shier, M. y Graham, J. (2011). Work-related factors that impact social work practitioners subjective well-being: Well-being in the workplace. Journal of Social Work, 11 (4), 402-421.

Spontón, C., Medrano, L., Maffel, L., Spontón, M., y Castellano, E. (2012). Validación del cuestionario de engagement Uwes a la población de trabajadores de Córdoba, Argentina. Liberabit, Revista de Psicología, 47-154.

Wheeler, K. G., (2002). Cultural Values in relation to equity sensitivity within and across cultures. Journal of Managerial Psychology, 17 (7), 612- 627. 